\title{
Iconic Tissue Level meets Immediacy
}

Straumann is delighted to have launched the latest generation of implants in a long line of clinically-proven and industry-leading products. The new TLX implant system is the perfect solution for clinicians looking to reach new heights in practice.

It has been designed to mimic the natural anatomy, respecting the hard and soft tissue for optimal aesthetics. The innovative system combines the benefits of a tissue level connection

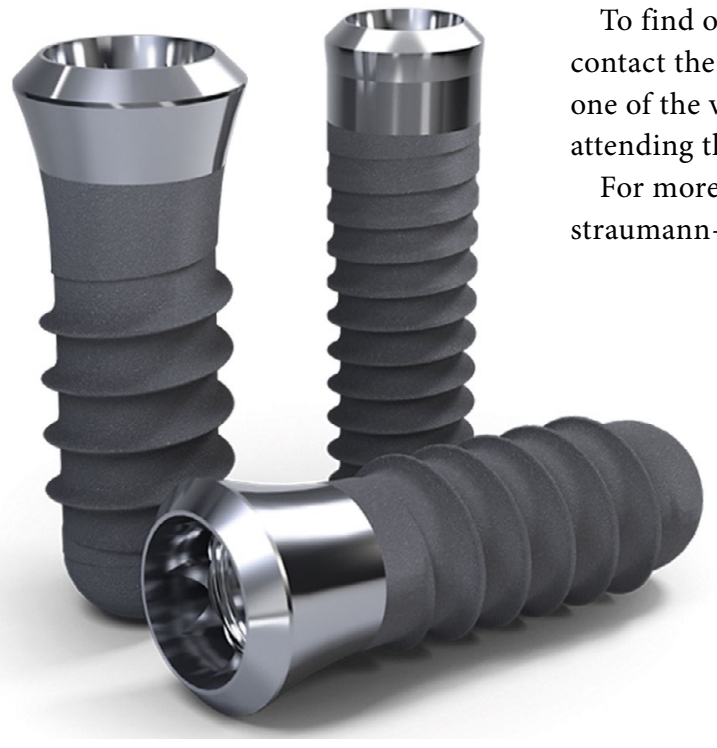

with the stability needed for immediacy and is an excellent solution for all other indications to suit the dentist's preferred treatment protocol - ranging from immediate to conventional placement and loading.

Helping patients restore their smiles and prevent resorption of both the bone and soft tissue. The TLX even uses the same drill set and TorcFit as the Straumann BLX bone level implant for professional workflow efficiency.

o find out more, visit the website,

contact the team or come and see us at the various live events we'll be ding this year! uk.co/tlx.

\section{Take the easy option!}

Managing your
air supply in
practice can be
time-consuming
and lead to
unnecessary
stress. So, why
not entrust your
air supply to
the experts at
DENTALAIR
UTILITIES?
With our Air to
the Chair option,
you receive installation of a brand new,
oil-free air supply that provides medical
grade air alongside the following
benefits, all for $£ 5$ per week per chair:
$\rightarrow$ All service costs
$\rightarrow$ Complete 24 -hour back-up support
$\rightarrow$ A PSSR written scheme and air quality
certification for CQC inspection
purposes.
We also have an option for professionals
that want to keep their existing air supply
- Air Care. This policy gives you all the
same benefits as Air to the Chair including
access to our world-class customer service
and support.

\section{In-practice trial kit launched}

Philips Oral Healthcare is constantly innovating to evolve its oral care solutions to make its Sonicare range more accessible to a wider range of patients, and its stated ambition is that one in ten consumers' daily routine is elevated by a Philips Sonicare toothbrush.

Whether at the start of an improved oral hygiene journey or looking for ways to upgrade from a manual approach, professional recommendation is a significant trigger for millions of dental patients. However, professionals need the right tools to be able to offer their patients a proactive as opposed to a reactive approach to transitioning to a powered brush.

To help with this goal Philips has introduced an in-practice Sonicare trial unit which allows patients to experience the unique sonic toothbrush feeling, risk free, as part of their appointment and take away a free brush head.
The new in-practice trial kit includes everything dental professionals need to help patients experience a new level of clean, coach them and address possible concerns they may have - and all this without the pressure of the patient having to make a purchase there and then.

The Sonicare starter kit which contains one ProtectiveClean 4700 power handle, 60 premium brush heads (30x plaque and $30 \mathrm{x}$ gum), 60 handle sleeves, 60 brush head bags, and 1 charger base, along with disinfection advice. This provides dental professionals with a tool to allow them to initiate a proactive conversation about manual-topower toothbrush conversion.

The Sonicare ProtectiveClean toothbrush features all the core elements which make the Sonicare brush so effective - but one of its primary attractions is its variable intensity settings which makes it so appealing for patients transitioning from a manual toothbrush. This allows dental professionals to show patients how to tune it up or down as they get used to the sonic sensation and to customise their brushing experience.

The slim handle design is also lightweight and easy to manoeuvre for patients who may only have used a manual brush. All Philips Sonicare brush heads are also recyclable through the company's Terracycle scheme.

To find out more about the in-practice trial kit and to sign up contact dental.sales.support@ philips.com.

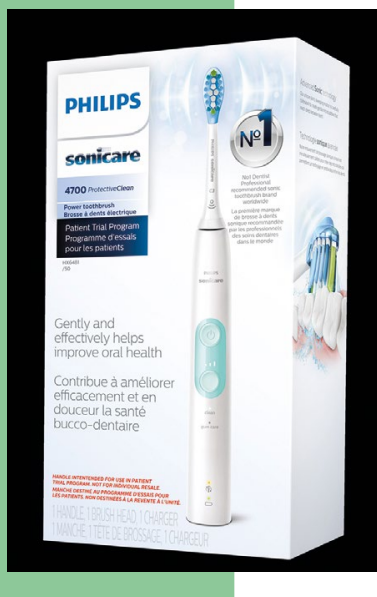

